\title{
Relevance of depth resolution for cerebral blood flow monitoring by near-infrared spectroscopic bolus tracking during cardiopulmonary bypass
}

Jens Steinbrink, PhD, ${ }^{a}$ Thomas Fischer, $\mathrm{MD},{ }^{\mathrm{c}}$ Hermann Kuppe, MD, ${ }^{\mathrm{c}}$ Roland Hetzer, MD, ${ }^{\mathrm{d}}$ Kamil Uludag, $\mathrm{PhD},{ }^{\mathrm{a}}$ Hellmuth Obrig, $\mathrm{MD}^{\mathrm{a}}$ and Wolfgang $\mathrm{M}$. Kuebler, $\mathrm{MD}^{\mathrm{b}, \mathrm{c}}$

From the Clinic of Neurology and Institute of Physiology, ${ }^{\mathrm{b}}$ Charité-Universitaetsmedizin and the Departments of Anesthesi$\operatorname{ology}^{\mathrm{c}}$ and Cardiothoracic and Vascular Surgery, ${ }^{\mathrm{d}}$ German Heart Institute, Berlin, Germany.

Supported in part by the Bundesministerium für Bildung und Forschung (BMBF) and the "Europäischer Fond für regionale Entwicklung" (EFRE)

Received for publication Feb 3, 2006; revisions received May 8, 2006; accepted for publication May 18, 2006.

Address for reprints: Jens Steinbrink, Charité University Hospital, Clinic of Neurology, Schumannstrasse 20/21, 10098 Berlin, Germany (E-mail: jens.steinbrink@charite.de).

J Thorac Cardiovasc Surg 2006;132:1172-8

$0022-5223 / \$ 32.00$

Copyright (๑) 2006 by The American Association for Thoracic Surgery

doi:10.1016/j.jtcvs.2006.05.065
Objective: Noninvasive near-infrared spectroscopy (NIRS) is increasingly used to monitor cerebral oxygenation and blood flow status, which is also of high relevance during cardiovascular surgical interventions with cardiopulmonary bypass. Contamination of the cerebral signal by contamination from overlaying extracerebral tissue, however, has been proposed to reduce sensitivity and cerebral selectivity of this promising technique.

Methods: We evaluated a novel depth-resolved approach for the determination of cerebral hemodynamics by near-infrared spectroscopic tracking of intravenously administered indocyanine green boluses. A frequency domain technique was applied, allowing simultaneous determination of light absorption changes and time of flight of single photons and enabling the differentiation between extracerebral and intracerebral tracer kinetics. Depth-resolved near-infrared spectroscopy was tested in 4 patients undergoing cardiopulmonary bypass and compared with data derived by conventional continuous-wave near-infrared spectroscopy.

Results: Depth resolution extracted the differential responses of extracerebral and intracerebral blood vessels from near-infrared bolus tracking signals. Postoperative blood flow indices derived from the intracerebral time course exceeded preoperative values by $1.5 \pm 0.2$ times, indicating a significant increase of cerebral blood flow not detectable by conventional near-infrared spectroscopy.

Conclusion: The depth-resolved approach provides additional and relevant data for the interpretation of intraoperative cerebral perfusion during cardiothoracic surgery. The validity of this approach for patients with preexisting risk factors for cerebral hypoperfusion remains to be determined in larger clinical trials.

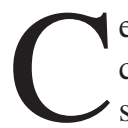
erebral injury, neurologic deficits, and cognitive impairment are major causes of intraoperative and perioperative morbidity and mortality in cardiac surgery with cardiopulmonary bypass (CPB). Prospective studies report a $3 \%$ incidence of stroke in patients undergoing coronary artery bypass grafting ${ }^{1}$ and an $8 \%$ rate for isolated valve surgery. ${ }^{2}$ The reported incidence of neurologic deficits after coronary artery bypass grafting ranges between $0.4 \%$ and $80 \%$, depending on definition of deficit and patient selection. ${ }^{3,4}$ Suggested mechanisms underlying neurologic damage during CPB comprise complete or incomplete cerebral ischemia, hypoxia, and embolization ${ }^{5}$ and therefore stress the need for monitoring of cerebral blood flow (CBF) and oxygenation. In contrast to computed tomographic and magnetic resonance imaging techniques, near-infrared spectroscopy (NIRS) facilitates noninvasive intraoperative and perioperative monitoring and has therefore been applied to measure cerebral tissue oxygenation during CPB. ${ }^{6,7}$ In addition, the combination of NIRS with the administration of the light absorbing intravascular 


\author{
Abbreviations and Acronyms \\ $\Delta \mathrm{c}_{\mathrm{ICG}}=$ change in indocyanine concentration \\ $\Delta \mu_{\mathrm{a}}=$ absorption change \\ $\mathrm{BFI}=$ blood flow index \\ $\mathrm{CBF}=$ cerebral blood flow \\ $\mathrm{CPB}=$ cardiopulmonary bypass \\ ICG = indocyanine green \\ NIRS $=$ near-infrared spectroscopy
}

dye indocyanine green (ICG) has been proposed for monitoring of regional cerebral perfusion. ${ }^{8-10}$

By applying laser Doppler flowmetry, a perfusion measurement without contrast agent has very recently been proposed. ${ }^{11}$ The advantages of NIRS are low cost and versatility; however, it is a major shortcoming that conventional NIRS cannot differentiate between absorption changes in cerebral and extracerebral tissue. ${ }^{12}$ Because of the noninvasive fixation of emitter and sensor probes (optodes) on the intact scalp, the detected signal is derived from cerebral structures as well as from extracerebral tissue (bone and skin). ${ }^{13}$ This consideration is of particular impact during $\mathrm{CPB}$, since intracerebral and extracerebral blood vessels, and thus blood flow and oxygenation, will respond differently to cardiotechnical and pharmacologic interventions. (1) Vascular autoregulation and $\mathrm{PaCO}_{2}$ vasoreactivity stabilize CBF but not extracerebral blood flow. (2) The effects of nonpulsatile flow during $\mathrm{CPB}$ on vascular tone and reactivity differ between different compartments. ${ }^{14}$ (3) Adrenergic innervation and vasoconstrictive response are predominantly confined to the extracerebral, not the intracerebral, compartment. ${ }^{15}$ (4) Finally, mild to moderate intraoperative hypothermia will affect $\mathrm{CBF}$ and extracerebral blood flow to different extents. ${ }^{16}$

To address these issues, we have recently proposed a new NIRS method, depth-resolved NIRS, which is the first to allow differentiation between intracerebral and extracerebral absorption changes. Note that commercial systems currently do not include this option. This method relies on the determination of the times of flight of photons, which can be measured by frequency or time domain techniques. Whereas intensity changes are strongly influenced by extracerebral absorption changes, alterations in the mean time of flight of photons more strongly depend on absorption changes in the depth of the tissue. ${ }^{17}$ Here we have applied a frequency domain approach, assessing attenuation and the mean time of flight of the photons to differentiate between $\mathrm{CBF}$ and extracerebral blood flow characteristics in patients undergoing $\mathrm{CPB}$.

\section{Methods \\ Patient Characteristics}

Depth-resolved NIRS was applied to monitor intraoperative CBF in 4 patients undergoing heart surgery with alpha-stat CPB. Procedural details are given in Table 1 . The trial was approved by the ethical committee of the Charité University Hospital, and informed consent was received from the patients.

\section{NIRS and Data Acquisition}

NIRS was performed with a commercially available frequency domain NIRS system (ISS Inc., Champaign, Ill) To maximize signal-to-noise ratio, only 1 of the available 16 laser diodes of the system was used at a wavelength of $810 \mathrm{~nm}$. The optical emitterdetector pair of the NIRS system was placed on the forehead $7 \mathrm{~cm}$ above the eye line, with a source-detector separation of $3 \mathrm{~cm}$.

Depth-resolved NIRS bolus tracking was performed during three phases of the surgery: (1) intra-operatively after thoracotomy and before the start of CPB, (2) during CPB and aortic clamping, and (3) after aortic declamping and cessation of CPB. At each time point, repeated injections (from 2-5) of ICG boluses (ICG-Pulsion; Pulsion Medical Systems, Munich, Germany) were administered through a central venous line. To allow NIRS signals to return to baseline between subsequent injections, the interval between the boluses was at least 4 minutes. Each bolus consisted of $5 \mathrm{mg}$ ICG dissolved in $3 \mathrm{~mL}$ water. Because of its high binding to plasma proteins, ICG predominantly distributes in the intravascular compartment. Its absorption peak at $805 \mathrm{~nm}$ allows the monitoring of its passage through the cerebral vasculature by NIRS and the calculation of hemodynamic parameters from the indicator dilution curves obtained. Each patient received 45 to $60 \mathrm{mg}$ ICG, which is safe and far below the maximum daily dose of $5 \mathrm{mg} / \mathrm{kg}$ body weight. No side effects of ICG were observed or have been reported in the literature.

\section{Data Analysis}

The frequency domain NIRS system used here supplies light, which is amplitude modulated at a frequency of $110 \mathrm{MHz}$ and can thus return three parameters: (1) the amplitude of the modulated intensity,

TABLE 1. Patient and procedural characteristics

\begin{tabular}{|c|c|c|c|c|}
\hline Characteristic & Patient A & Patient B & Patient C & Patient D \\
\hline Age (y) & 71 & 61 & 78 & 66 \\
\hline Sex & Female & Male & Male & Male \\
\hline BMI $\left(\mathrm{kg} / \mathrm{m}^{2}\right)$ & 21.8 & 33.7 & 22.3 & 23.2 \\
\hline Diagnosis & 3-vessel CAD & 3-vessel CAD & 3-vessel CAD & AV stenosis \\
\hline Procedure & Triple CABG & Triple CABG & Triple CABG & AV replacement \\
\hline CPB time (min) & 92 & 93 & 113 & 76 \\
\hline
\end{tabular}

$B M I$, Body mass index; $C A D$, coronary artery disease; $A V$, aortic valve; $C A B G$, coronary artery bypass grafting. 
TABLE 2. Intraoperative hemodynamics, blood gases, and body temperature

\begin{tabular}{lccc}
\hline & Before CPB & During CPB & After CPB \\
\hline MAP $(\mathrm{mm} \mathrm{Hg})$ & $78.8 \pm 5.3$ & $63.8 \pm 2.1$ & $70.4 \pm 3.8$ \\
$\mathrm{HR}$ (beats/min) & $88.8 \pm 12.5$ & 0 & $88.8 \pm 4.7$ \\
$\mathrm{Hb}(\mathrm{g} / 100 \mathrm{~mL})$ & $12.2 \pm 1.0$ & $8.0 \pm 0.5$ & $9.2 \pm 0.4$ \\
$\mathrm{PaO}_{2}(\mathrm{~mm} \mathrm{Hg})$ & $315.6 \pm 62.1$ & $172.6 \pm 27.8$ & $227.5 \pm 30.6$ \\
$\mathrm{Paco}_{2}(\mathrm{~mm} \mathrm{Hg})$ & $34.1 \pm 1.3$ & $36.7 \pm 1.2$ & $36.6 \pm 2.5$ \\
Temperature $^{*}\left({ }^{\circ} \mathrm{C}\right)$ & $36.0 \pm 0.2$ & $32.6 \pm 0.5$ & $36.3 \pm 0.1$
\end{tabular}

Data are mean \pm SD for 4 subjects. $C P B$, Cardiopulmonary bypass, $M A P$, mean arterial pressure; $H R$, heart rate; $H b$, hemoglobin. *Measured with an intravesical thermistor catheter.

(2) the phase of the modulated intensity, and (3) the intensity of the nonmodulated light. By analyzing changes in phase and the unmodulated intensity, we have previously been able to separate absorption changes in extracerebral scalp from those in brain. ${ }^{17}$ The depth discrimination procedure returns changes $\left(\Delta \mu_{\mathrm{a}}\right)$ in the brain $\left(\Delta \mu_{\text {a,brain }}\right)$ and the extracerebral $\left(\Delta \mu_{\mathrm{a}, \text { extra }}\right)$ compartments. ${ }^{17}$

The changes in the absorption coefficients measured can be transformed into concentration changes of ICG by using tabulated extinction coefficients $(\varepsilon) .{ }^{18} \mathrm{We}$ used $\Delta \mathrm{c}_{\mathrm{ICG}}=\Delta \mu_{\mathrm{a} \text {,brain }} / \varepsilon$, with $1 / \varepsilon$ approximately $42 \mathrm{mg} \cdot \mathrm{mm} / \mathrm{L}$ at $810 \mathrm{~nm}$, to calculate changes in ICG concentration $\left(\Delta c_{I C G}\right)$ in tissue. Thus the depth-resolved approach applied in this study yields time courses for absorption or concentration changes in the extracerebral and intracerebral compartments. From the time course of these changes, hemodynamic blood flow parameters can be extracted by adaptation of algorithms derived from indicator dilution techniques. Here we applied our recently reported and validated blood flow index (BFI) to analyze the effect of depth resolution and the contribution of extracerebral tissue to the NIRS signal during CPB. BFI is defined as $\Delta \mathrm{c}_{10 \%-90 \%}$ / $\mathrm{t}_{10 \%-90 \%}$, where $\Delta c_{10 \%-90 \%}$ is the change between $10 \%$ and $90 \%$ of the maximum concentration change and $t_{10 \%-90 \%}$ is the corresponding rise time. ${ }^{17}$ Similar to blood flow parameters determined from bolus tracking techniques with magnetic resonance imaging, the BFI is a relative parameter; thus its baseline value is arbitrary.

Note that although the hardware for the proposed approach is commercially available, the data analysis was performed off line. The steps of the algorithm are easy to implement, however, and we hope that an on-line version will be provided by the system developers. The signal of each injection of ICG could be clearly identified, even during the surgical procedure. Only maneuvers with a very strong motion of the patient's body resulted in artifacts, which could be identified by multiple and rapid signal changes.

\section{Statistics}

Unless stated otherwise, all data are presented as mean \pm SD. Data were analyzed for significant changes between values determined before and after CPB by Mann-Whitney $U$ test.

\section{Results}

Intraoperative data on patient hemodynamics, blood gases, and intravesically determined body temperature before, during, and after CPB are summarized in Table 2.

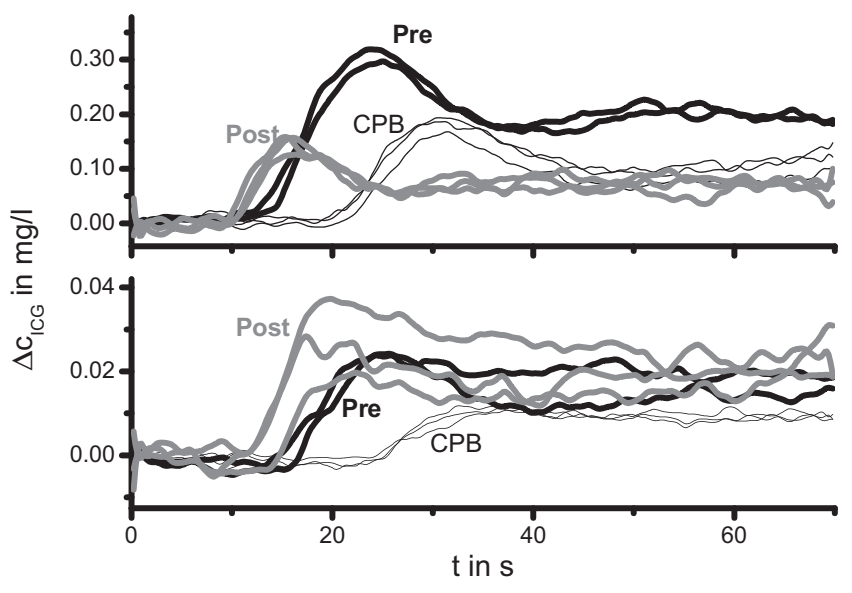

Figure 1. Representative tracings of several intravenously administered indocyanine green boluses monitored by depth-resolved near-infrared spectroscopy in intracerebral (upper panel) and extracerebral (lower panel) compartments before (pre), during (CPB), and after (post) cardiopulmonary bypass in patient $\mathrm{C}$. Note low variance of intracerebral versus extracerebral signal. $\Delta c_{I C G}$ Change in indocyanine green concentration.

During the three phases of the surgical intervention defined in the Methods section, boluses of ICG were administered intravenously, and passage through the patient's head was monitored by cranial depth-resolved NIRS. Figure 1 provides representative tracings of ICG absorption in the intracerebral and extracerebral compartments as determined in patient C. For each time point, two to three time courses derived from different bolus injections are shown. During the operative procedure, the time courses of both intracerebral and extracerebral bolus passages changed considerably. Intracerebral signals showed a high reproducibility at each time point during repetitive bolus injections, whereas extracerebral signals fluctuated considerably between subsequent measurements.

The recorded ICG tracer kinetics before, during, and after CPB are given for each patient in Figure 2. Individual time courses were derived from averaging the tracer curves from two to five consecutive bolus injections. Before CPB, patients $\mathrm{A}$ through $\mathrm{C}$ showed a pronounced peak in the intracerebral ICG time course. In line with the expectation of a higher CBF velocity relative to a slower flow velocity in scalp and skull, the bolus arrived earlier in the intracerebral compartment. During $\mathrm{CPB}$, the intracerebral tracer profile was largely stable in all 4 patients, whereas the peak in the extracerebral compartment was markedly reduced and delayed. After CPB, the latency of the ICG bolus in the intracerebral compartment was reduced and the upward slope steepened, indicating a marked reduction in the transit time of the bolus. Accordingly, 3 of the 4 patients showed a significant increase in BFI, as determined from the intra- 

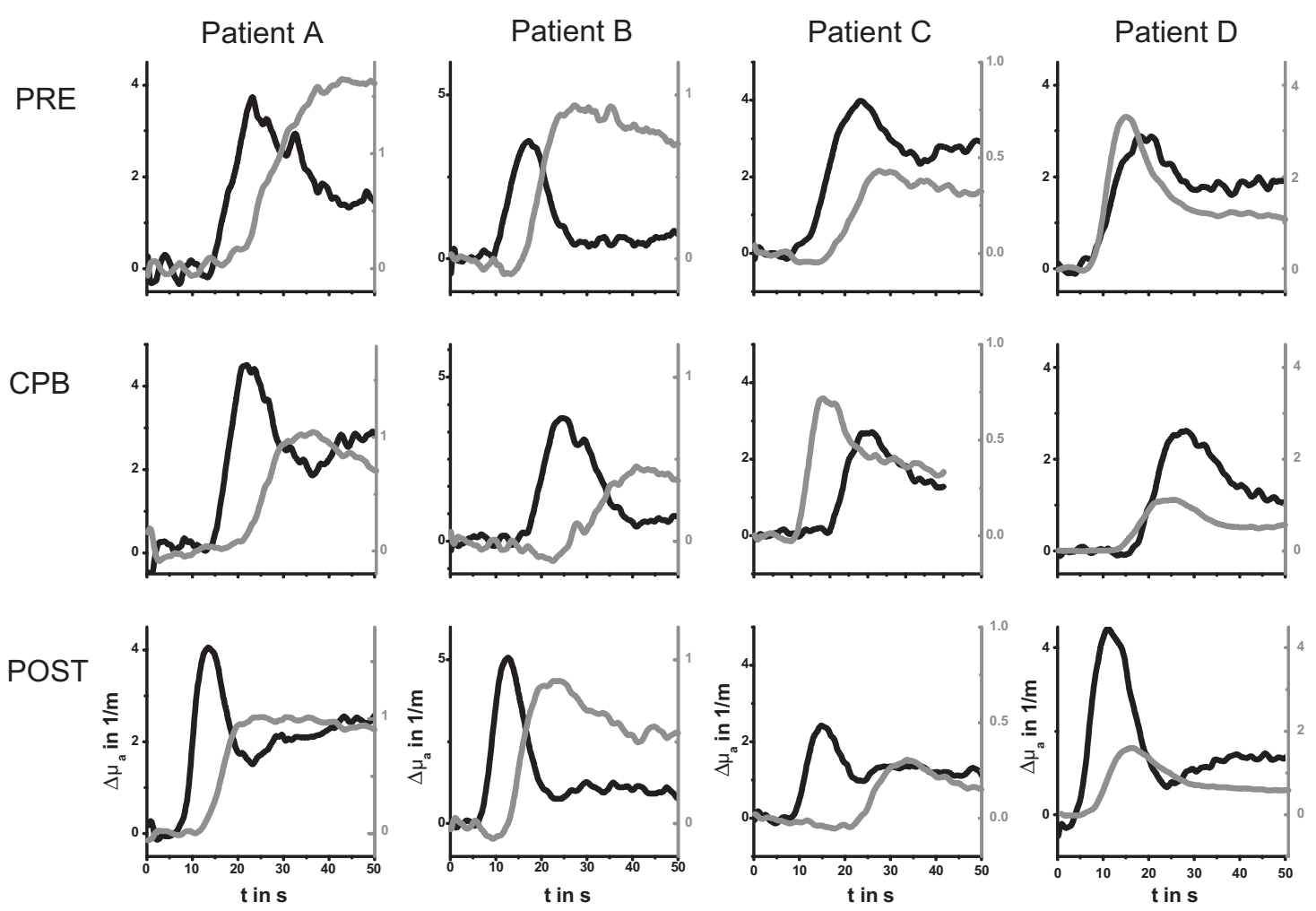

Figure 2. Averaged intracerebral (black) and extracerebral (gray) tracer kinetics of intravenous indocyanine green boluses as monitored by depth-resolved near-infrared spectroscopy before (PRE), during (CPB), and after (POST) cardiopulmonary bypass in 4 patients. To account for large intersubject variations, range of $y$-axis is kept fixed for all three phases but is different for all 4 patients. $\Delta \mu_{a}$, Change in absorption.

cerebral ICG-bolus time courses after CPB relative to measurements before CPB (Figure 3, A).

To compare depth-resolved NIRS bolus tracking with conventional approaches by a continuous-wave NIRS approach without depth resolution, we monitored ICG kinetics by intensity measurements only and calculated BFI values from these signal curves, thus comprising both intracerebral and extracerebral compartments. Figure 4 demonstrates the relevance of a depth-resolved NIRS approach. Normalized time courses for the ICG bolus are given for either a depth-resolved or a continuous wave (conventional) NIRS approach. In contrast to the depth-resolved approach differentiating between intracerebral and extracerebral compartments, conventional techniques with mere intensity measurements yield indicator tracing curves, which are a weighted average of both signals. Figure 4 demonstrates that the upward slope of the ICG curve is strongly influenced by the delayed arrival of the extracerebral as compared with the intracerebral bolus. This results in a prolongation of the rise

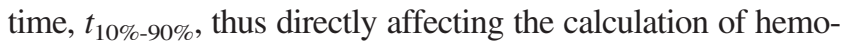
dynamic parameters such as the BFI. Thus BFIs calculated from a conventional approach no longer reveal altered $\mathrm{CBF}$ after CPB relative to measurements before CPB (Figure 3, B). In other words, extracerebral blood flow strongly contaminates conventional continuous-wave NIRS bolus tracings, thus potentially obscuring the signal of interest, $\mathrm{CBF}$.

\section{Discussion}

Our study demonstrates the feasibility of a new depthresolved NIRS bolus tracking technique during cardiothoracic surgery. This novel approach extracts additional data on cerebral perfusion from ICG bolus curves and yields reproducible results that are more reliable than those of conventional NIRS approaches, which are highly sensitive to extracerebral contamination. Thus this technique renders NIRS a potentially valuable tool to further investigate specific risk factors (eg, concomitant cerebrovascular disease) for focal or global ischemia during heart surgery. Although this goal requires larger clinically oriented studies, we here demonstrate methodologic advances to minimize potential extracerebral contamination of the signal. For the approach presented here, discrimination between superficial and deep tissue layers is achieved by a frequency domain approach in which the differential influences of intracerebral and extra- 

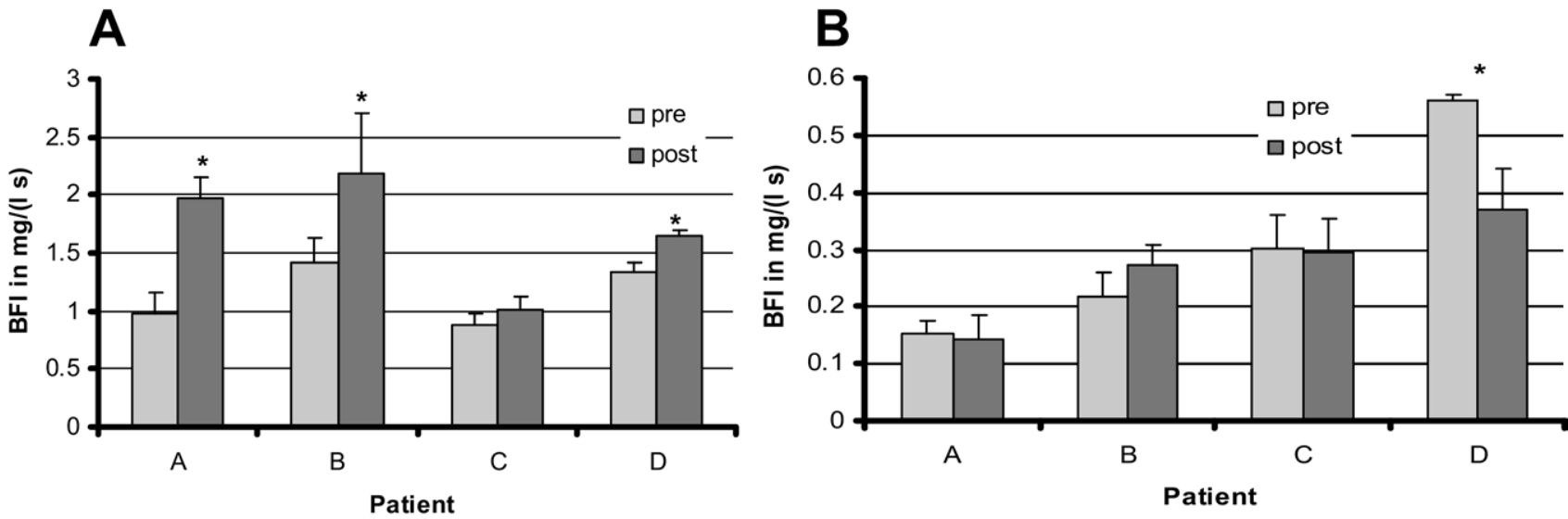

Figure 3. Mean blood flow index (BFI) determined from multiple indocyanine green bolus curves before (pre) and after (post) cardiopulmonary bypass. Asterisks indicate significant changes according to Mann-Whitney $U$ test in individual subjects. (A) In case of depth-resolved analysis, significant $1.5 \pm 0.3$ times (mean \pm SD) increase in blood flow index was detected in 4 subjects. (B) By conventional analysis, no significant change in blood flow index was observed.

cerebral layers on attenuation changes and mean transit times of photons is estimated by a Monte Carlo simulation. In 4 patients undergoing $\mathrm{CPB}$, we have demonstrated the approach to yield more robust intracerebral indicator dilution curves and to unravel additional information on $\mathrm{CBF}$

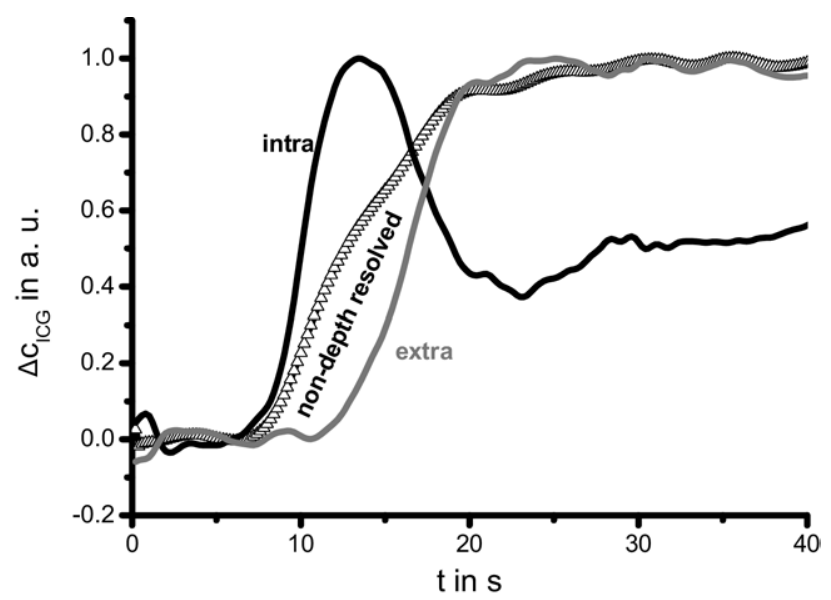

Figure 4. Comparison of indocyanine green tracer kinetics detected by depth-resolved and conventional near-infrared spectroscopy. Representative tracings are derived from averaged bolus kinetics recorded in patient $A$ after cardiopulmonary bypass. Normalized indocyanine green concentration is given for intracerebral (black) and extracerebral (gray) indocyanine green kinetics, as recorded by depth-resolved near-infrared spectroscopy, and a conventional signal curve as would be recorded by continuous-wave near-infrared spectroscopy without depth resolution (open triangles). $\Delta c_{I c G^{\prime}}$ Change in indocyanine green concentration; a.u., arbitrary units. not accessible by conventional NIRS techniques. Specifically, depth-resolved NIRS bolus tracking revealed (1) that the $\mathrm{CBF}$ was largely maintained during $\mathrm{CPB}$, whereas the extracerebral blood flow was markedly attenuated, and (2) that $\mathrm{CBF}$ was higher after $\mathrm{CPB}$ than before $\mathrm{CPB}$ in 3 of 4 patients. A conventional continuous-wave NIRS will not be able to detect such changes. Depth-resolved NIRS is therefore an important novel extension of the methodology for noninvasive intraoperative monitoring of $\mathrm{CBF}$.

\section{Perspectives and Limitations of Depth-resolved NIRS} Bolus Tracking

NIRS tracking of intravascular tracers has previously been proposed as a technique to monitor CBF. ${ }^{9,10,19,20}$ Although spatial specificity is much lower with the currently available technology, the noninvasive approach is an intraoperatively applicable extension to blood flow imaging with computed tomography and magnetic resonance imaging. Intraoperative monitoring facilitates immediate countermeasures to ensure adequate $\mathrm{CBF}$ and oxygenation during $\mathrm{CPB}$.

Different algorithms have been proposed to yield a sensitive and reproducible detection of intracerebral tracer kinetics and to acquire a quantitative or semiquantitative measure of CBF by NIRS. Although various studies demonstrated feasibility and reproducibility of CBF measurements by the Fick principle, ${ }^{9,19}$ the derived data could not be correlated in a study with CBF values determined by the radioactive microsphere technique,$^{21}$ the criterion standard for CBF measurements. BFI, a semiquantitative parameter derived from fluorescence flowmetry, was validated against radioactive microspheres in pigs, ${ }^{17}$ and its reproducibility has been demonstrated in several patient studies. ${ }^{5,12}$ 
The primary requirement for NIRS-based determination of $\mathrm{CBF}$, however, the sensitive and reproducible detection of intracerebral tracer kinetics, is not unrestrictedly warranted in conventional continuous-wave NIRS. Because the regional distribution of the tracer dye is not limited to the intracerebral compartment, contamination from extracerebral blood vessels may alter the detected signal curves and impair accurate detection of cerebral hemodynamics.

We recently proposed a novel approach to allow differentiation between intracerebral and extracerebral signals by use of depth-resolved NIRS. ${ }^{22,23}$ This technique can be applied for bolus tracking of intravascular tracer dyes with light-absorbing properties in the near-infrared range, such as ICG. ${ }^{17,22,24}$ In principle, the method relies on the simultaneous determination of the time of flight of photons, which can be measured by frequency or time domain techniques, and of changes in intensity of the light back scattered from the tissue. Whereas intensity changes are strongly affected by variations in extracerebral light absorption, changes in the mean transit time of photons are more strongly influenced by light absorption in the depth of the tissue. ${ }^{17} \mathrm{~A}$ potential limitation of this novel approach lies in its dependence on the different sensitivity factors $l$ and $m$ for light absorption changes in the intracerebral and extracerebral compartments, which are estimated from Monte Carlo simulations. ${ }^{23}$ Accordingly, model assumptions such as thickness of skin and skull layers or optical homogeneity of the tissue directly influence the applied algorithms for depth resolution of the recorded signals. This will be a principal problem if quantitative measures are required, whereas qualitative measurements show relative robustness toward plausible variations of the assumed thickness of the different layers.

In this study, intracerebral tracer kinetics recorded by depth-resolved NIRS showed a higher degree of reproducibility than did extracerebral signals and revealed significant changes in intracerebral bolus kinetics that were not detectable by conventional NIRS. The proposed method thus increases both robustness and sensitivity of CBF monitoring by NIRS and is therefore a relevant advance toward reliable monitoring of $\mathrm{CBF}$ during $\mathrm{CPB}$ and cardiovascular surgical procedures.

\section{Intracranial and Extracranial Blood Flow Profiles}

Before CPB, 3 of 4 patients showed earlier arrival of the tracer bolus in the intracerebral than the extracerebral compartment, which is in good agreement with the physiologic expectation of higher blood flow velocities in brain than in skin. Whereas mean capillary blood flow velocity in human skin varies between 0.15 and $0.25 \mathrm{~mm} / \mathrm{s},{ }^{25}$ velocities in capillaries of the central nervous system (eg, the olfactory bulb glomeruli) range between 0.2 and $1.0 \mathrm{~mm} / \mathrm{s}$, with a mean of $0.57 \mathrm{~mm} / \mathrm{s}^{26}$
Whereas pre-CPB and post-CBF tracer kinetics can be directly compared by calculation of BFI, tracer arrival time and bolus dilution will be altered during $\mathrm{CPB}$ as a result of bolus passage through the extracorporeal circuit. Thus evaluation of tracer kinetics during CPB is limited to intraindividual comparisons between intracerebral and extracerebral tracer kinetics. We were able to show the intracerebral tracer profile to be largely conserved, although tracer kinetics derived from the extracerebral compartment showed reduced amplitude and a delayed and diminished upslope relative to pre-CPB recordings. This indicates a relative reduction in extracerebral blood volume and blood flow velocity relative to the intracerebral compartment. This differential regulation of intracerebral and extracerebral blood vessels can be attributed to the differential response to adrenergic stimulation, temperature, or $\mathrm{PaCO}_{2}$ variations. ${ }^{15}$

After $\mathrm{CPB}$, the profile of extracerebral tracer kinetics partially recovered, whereas intracerebral kinetics revealed a marked acceleration of bolus transit relative to pre-CPB measurements. This is indicated by a shortened bolus appearance time and a steeper upslope. In accordance with this notion, BFI increased significantly in 3 of 4 patients. This effect may be explained by the increased $\mathrm{PaCO} 2$ and reduced hematocrit ${ }^{27}$ after $\mathrm{CPB}$ relative to pre-CPB measurements. This finding is in accordance with experiments in pigs, in which cerebral vascular resistance was notably reduced 15 and 120 minutes after hypothermic CPB relative to baseline values. ${ }^{28}$ Moreover, patients without preexisting cerebrovascular disease have a markedly higher jugular bulb oxygen saturation after 60 minutes of normothermic CPB than before $\mathrm{CPB} .{ }^{29}$ In contrast, no increase in jugular bulb oxygen saturation was detected in 9 patients with preexisting stroke in the same study. Of note, patient $\mathrm{C}$ was the only patient in our study with a history of an ischemic stroke, 1 year before the operation, and also was the only patient in whom intracerebral BFI did not increase. This lack of response thus may indicate a preexisting cerebrovascular impairment not attributable to the intraoperative procedures.

In conclusion, the monitored intraoperative changes of the CBF and extracerebral blood flow profiles match physiologic and pathophysiologic response patterns yet would be undetectable by conventional NIRS approaches. Implementation of depth-resolving algorithms for tracer bolus tracking in frequency or time domain NIRS systems is thus mandatory to allow on-line differentiation between intracerebral and extracerebral components. Further studies are required to test the reliability of this novel approach in different clinical settings.

We are indebted to the cardiotechnical staff at the Deutsches Herzzentrum Berlin for their valuable support. 


\section{References}

1. Hogue CW Jr, Barzilai B, Pieper KS, Coombs LP, DeLong ER, Kouchoukos NT, et al. Sex differences in neurological outcomes and mortality after cardiac surgery: a Society of Thoracic Surgery national database report. Circulation. 2001;103:2133-7.

2. Wolman RL, Nussmeier NA, Aggarwal A, Kanchuger MS, Roach GW, Newman MF, et al. Cerebral injury after cardiac surgery: identification of a group at extraordinary risk. Multicenter Study of Perioperative Ischemia Research Group (McSPI) and the Ischemia Research Education Foundation (IREF) Investigators. Stroke. 1999;30: $514-22$.

3. Blauth CI, Arnold JV, Schulenberg WE, McCartney AC, Taylor KM. Cerebral microembolism during cardiopulmonary bypass. Retinal microvascular studies in vivo with fluorescein angiography. $J$ Thorac Cardiovasc Surg. 1988;95:668-76.

4. Roach GW, Kanchuger M, Mangano CM, Newman M, Nussmeier N, Wolman R, et al. Adverse cerebral outcomes after coronary bypass surgery. Multicenter Study of Perioperative Ischemia Research Group and the Ischemia Research and Education Foundation Investigators. N Engl J Med. 1996;335:1857-63.

5. Taggart DP, Westaby S. Neurological and cognitive disorders after coronary artery bypass grafting. Curr Opin Cardiol. 2001;16:271-6.

6. Hoffman GM, Stuth EA, Jaquiss RD, Vanderwal PL, Staudt SR, Troshynski TJ, et al. Changes in cerebral and somatic oxygenation during stage 1 palliation of hypoplastic left heart syndrome using continuous regional cerebral perfusion. J Thorac Cardiovasc Surg. 2004; 127:223-33.

7. Nollert G, Jonas RA, Reichart B. Optimizing cerebral oxygenation during cardiac surgery: a review of experimental and clinical investigations with near infrared spectrophotometry. Thorac Cardiovasc Surg. 2000;48:247-53.

8. Kuebler WM, Sckell A, Habler O, Kleen M, Kuhnle GE, Welte M, et al. Noninvasive measurement of regional cerebral blood flow by near-infrared spectroscopy and indocyanine green. J Cereb Blood Flow Metab. 1998;18:445-56.

9. Roberts I, Fallon P, Kirkham FJ, Lloyd-Thomas A, Cooper C, Maynard R, et al. Estimation of cerebral blood flow with near infrared spectroscopy and indocyanine green. Lancet. 1993;342:1425.

10. Terborg C, Bramer S, Harscher S, Simon M, Witte OW. Bedside assessment of cerebral perfusion reductions in patients with acute ischaemic stroke by near-infrared spectroscopy and indocyanine green. J Neurol Neurosurg Psychiatry. 2004;75:38-42.

11. Durduran T, Yu G, Burnett MG, Detre JA, Greenberg JH, Wang J, et al. Diffuse optical measurement of blood flow, blood oxygenation, and metabolism in a human brain during sensorimotor cortex activation. Opt Lett. 2004;29:1766-8.

12. Germon TJ, Young AE, Nelson RJ. Near-infrared spectroscopy. J Neurosurg. 1995;83:1111-2.

13. Young AE, Germon TJ, Barnett NJ, Manara AR, Nelson RJ. Behaviour of near-infrared light in the adult human head: implications for clinical near-infrared spectroscopy. Br J Anaesth. 2000;84:38-42.

14. Ohnishi Y, Hu QH, Yamaguchi S, Kuro M, Niimi H. Cerebral microcirculatory changes in rat with a cardiopulmonary bypass using fluorescence videomicroscopy. Clin Hemorheol Microcirc. 2002;26:15-26.
15. Brown CG, Robinson LA, Jenkins J, Bowman K, Schlaifer J, Werman $\mathrm{H}$, et al. The effect of norepinephrine versus epinephrine on regional cerebral blood flow during cardiopulmonary resuscitation. Am J Emerg Med. 1989;7:278-82.

16. Su JY, Amory DW, Sands MP, Mohri H. Effects of circulatory arrest and rewarming on regional blood flow during surface-induced hypothermia. Am Heart J. 1980;100:332-40.

17. Kohl-Bareis M, Obrig H, Steinbrink J, Malak J, Uludag K, Villringer A. Noninvasive monitoring of cerebral blood flow by a dye bolus method: separation of brain from skin and skull signals. J Biomed Opt. 2002;7:464-70.

18. Landsman ML, Kwant G, Mook GA, Zijlstra WG. Light-absorbing properties, stability, and spectral stabilization of indocyanine green. J Appl Physiol. 1976;40:575-83.

19. Keller E, Nadler A, Alkadhi H, Kollias SS, Yonekawa Y, Niederer P. Noninvasive measurement of regional cerebral blood flow and regional cerebral blood volume by near-infrared spectroscopy and indocyanine green dye dilution. Neuroimage. 2003;20:828-39.

20. Kusaka T, Isobe K, Nagano K, Okubo K, Yasuda S, Kondo M, et al. Estimation of regional cerebral blood flow distribution in infants by near-infrared topography using indocyanine green. Neuroimage. 2001; 13:944-52.

21. Newton CR, Wilson DA, Gunnoe E, Wagner B, Cope M, Traystman RJ. Measurement of cerebral blood flow in dogs with near infrared spectroscopy in the reflectance mode is invalid. J Cereb Blood Flow Metab. 1997; 17:695-703.

22. Liebert A, Wabnitz H, Steinbrink J, Obrig H, Moller M, Macdonald R, et al. Time-resolved multidistance near-infrared spectroscopy of the adult head: intracerebral and extracerebral absorption changes from moments of distribution of times of flight of photons. Appl Opt. 2004;43:3037-47.

23. Steinbrink J, Wabnitz H, Obrig H, Villringer A, Rinneberg H. Determining changes in NIR absorption using a layered model of the human head. Phys Med Biol. 2001;46:879-96.

24. Ostergaard L, Weisskoff RM, Chesler DA, Gyldensted C, Rosen BR. High resolution measurement of cerebral blood flow using intravascular tracer bolus passages. Part I: mathematical approach and statistical analysis. Magn Reson Med. 1996;36:715-25.

25. Fagrell B, Intaglietta M, Ostergren J. Relative hematocrit in human skin capillaries and its relation to capillary blood flow velocity. $\mathrm{Mi}$ crovasc Res. 1980;20:327-35.

26. Chaigneau E, Oheim M, Audinat E, Charpak S. Two-photon imaging of capillary blood flow in olfactory bulb glomeruli. Proc Natl Acad Sci US A. 2003;100:13081-6.

27. Sakamoto T, Nollert GD, Zurakowski D, Soul J, Duebener LF, Sperling $\mathrm{J}$, et al. Hemodilution elevates cerebral blood flow and oxygen metabolism during cardiopulmonary bypass in piglets. Ann Thorac Surg. 2004;77:1656-63.

28. Strauch JT, Spielvogel D, Haldenwang PL, Zhang N, Weisz D, Bodian $\mathrm{CA}$, et al. Impact of hypothermic selective cerebral perfusion compared with hypothermic cardiopulmonary bypass on cerebral hemodynamics and metabolism. Eur J Cardiothorac Surg. 2003;24:807-16.

29. Kadoi Y, Saito S, Kawahara F, Goto F, Owada R, Fujita N. Jugular venous bulb oxygen saturation in patients with preexisting diabetes mellitus or stroke during normothermic cardiopulmonary bypass. Anesthesiology. 2000;92:1324-9. 